\title{
ARCHITECTURE FOR DISCRETE CONSTRUCTION COMPONENT TRACKING
}

\author{
Karen M. Furlani and William C. Stone \\ Construction Metrology and Automation Group \\ National Institute of Standards and Technology \\ Gaithersburg, Maryland 20899, USA \\ kfurlani@nist.govwilliam.stone@nist.gov
}

\begin{abstract}
Accurate and timely identification and tracking of construction components are critical to operating a well-managed and cost efficient construction project. Establishing standards to support identification and tracking technologies has the potential to enable the construction industry to seamlessly integrate work processes at the job-site. This paper discusses on-going research at NIST in the application of field sensors, portable computers, real-time high-precision global positioning, wireless communications, and construction project databases, to develop means for real-time component identification and tracking.
\end{abstract}

Keywords: component tracking, construction, global positioning system, metrology sensors, real-time tracking, RFID communications standards, wireless data transfer.

\section{INTRODUCTION}

Excessive amounts of time are spent on construction sites everyday locating and identifying components and sub-assemblies. A significant portion of this tracking process involves field workers searching lay-down areas and using paperbased tracking systems to identify objects and find particular components. This is true even with the present trend toward quantized "work package" delivery. Once an item has been located and properly identified, questions arise such as: Where does the item belong? How should it be installed? Is the final position and orientation correct? Developing the means for an automated "cradle-tograve" tracking system for components and subassemblies, from their arrival on a construction site through their final placement, will decrease dataentry errors and increase overall productivity.

Although many technologies exist to aid in the automation of component tracking systems, their uses are limited by a lack of construction industry standards supporting inter-operability between various hardware and software systems; for example, bar code and radio frequency sensors, global positioning systems, and wireless communications. Additionally, many products are not designed to function and survive in the hostile and dynamic environment of a typical construction site.
The Construction Metrology and Automation (CMA) Group at the National Institute of Standards and Technology (NIST) has initiated a project in Real-Time Construction Component Tracking to address the problem of identifying, registering, and tracking discrete construction components and subassemblies on a construction site. Discrete components in the context of the present discussion are manufactured, prefabricated singluar items (such as wide flange steel beams) which might logically carry an identification means (such as a bar code or RFID tag). The project objectives are to: 1) develop standards for part ID and tracking that the construction industry will adopt; 2) develop means for real-time tracking of sub-assemblies and components, and 3) develop standard means to wirelessly transmit that information to a construction project database; and, 4) to demonstrate the utility of these techniques on full-scale construction sites.

\section{PROJECT SCOPE}

The prototype component tracking system developed for this project integrates field sensors, portable computers, wireless communication, and real-time kinematic (RTK) global positioning system (GPS) equipment. Individual objects scheduled for arrival on the construction site are tagged at the 
fabricators using bar codes or radio-frequency transponders (RFID). The encoded information is scanned directly into a portable computer and wirelessly relayed to a remote project database. A database query returns graphical representations \{e.g. computer aided design (CAD) information, or virtual reality mark-up language (VRML) models $\}$ of scanned objects and additional information as appropriate. These models, coupled with userfriendly web browsing software, guide field workers through the acquisition of key fiducial points using scanning devices integrated with GPS technology to determine an object's position and orientation. The database then uses this data to track the instant state of the object.

Throughout the development of this tracking system, the project has focused on:

(1) The information content of the identification tag (e.g., manufacturer, part ID);

(2) Standards for marking components for position and attitude measurements;

(3) The wireless communications standards used to transmit the information; and

(4) Interface standards for metrology systems used for real-time tracking.

\section{SYSTEM DESCRIPTION}

\subsection{Sensing Technologies}

Two sensing technologies that support automated data entry are being investigated: Bar code and radio frequency identification (RFID).

The NIST project has made use of the "Edge" bar code scanning system from Aedex Inc.1., and "BarTender" label printing software from Seagull Scientific Systems with an Eltron TLP 2642 printer. The RFID system is the HT EV400 Hitag Proximity Evaluation Kit from Philips Semiconductors.

\subsubsection{Bar Code}

Bar code technology is based on a labeling system comprised of light and dark bars on printed labels [1]. Bar code symbology is the encoding scheme used to convert character data into a specific pattern of wide and narrow dark bars and light spaces. This project currently uses Code 39 , or Code

\footnotetext{
${ }^{1}$ Certain trade names or company products are mentioned in the text or identified in an illustration in order to adequately specify the experimental procedure and equipment used. In no case does such an identification imply recommendation or endorsement by the National Institute of Standards and Technology, nor does it imply that the products are necessarily the best available for the purpose.
}

3-of-9, symbology which contains the following 43 characters: Digits 0-9; capital letters A-Z; symbols \% $+\$ .-1 ;$ and the space character [3]. In addition to allowing variable length codes using both alphabetic and numeric characters, Code 39 was chosen for is overall robustness and reading accuracy supported by structural simplicity, self-checking and bi-directional reading [2]. Each Code 39 character is represented by nine elements: five bars and four spaces. The name Code 3-of-9 is derived from three of the nine elements being wide (the remaining six are narrow.)

\subsubsection{Bar Code Hardware}

Two types of hand-held bar-code reading devices are employed, a wand and a laser gun. Both use a low power visible red LED (Light Emitting Diode) as their light source and are constructed to work with the low power signals available from an RS232C port. The bar code "seen" by these readers is the light pattern reflected back to the photodetector, which converts the black bars and white spaces into a serial string of electrical signals.

The wand operates using power available from the RS232 port to emit a beam of light from the tip. The wand's tip must be in direct contact with a bar code to ensure a scan.

The laser gun uses an internal $9 \mathrm{~V}$ battery to sweep a laser beam across a bar code approximately 35 times/second. The optics in the scanner have a "depth of field" much larger than the quasi-contact wand device, enabling the gun readers to successfully scan bar codes up to approximately 70 $\mathrm{cm}$ - away depending upon the symbol density.

\subsubsection{Software}

Memory resident software extensions on the computer are used to decode the serial string of electrical signals from the scanned bar code into standard ASCII data. The software serves as a pseudo-keyboard for the computer -- to allow information from the scanner to enter the computer, as if typed by hand-.

\subsubsection{RFID}

Radio frequency identification (RFID) technology involves the use of a radio frequency reading/writing $(\mathrm{R} / \mathrm{W})$ station in conjunction with a transponder tag to enable two-way communication. The passive transponders used in this project contain an integrated circuit and antenna that are encapsulated to protect against impact and environmental elements. The $\mathrm{R} / \mathrm{W}$ station sends an $\mathrm{RF}$ carrier to the transponder, which is received by the antenna and converted to direct current (DC) to power the tag and start a communication session with 
the reader. The $\mathrm{R} / \mathrm{W}$ station converts the analog output from the transponder into digital format to feed to the computer.

Direct contact and direct line-of-sight are not required for accurate RFID readings. Passive tags can be read through a variety of non-metallic materials at distances up to approximately $80 \mathrm{~cm}$; however, metal can hamper RF tag operation by blocking and canceling the signal. Reading speeds are sufficient to acquire batches of tags at the average walking speed of a construction inspector. RFID tags have the further significant capability of storing up to several kilobits of information, a factor which can be of significant benefit for keeping local track of high value, high maintenance components (e.g. valves) in remote locations which require periodic servicing or calibration.

\subsection{Portable Computing}

We presently use an IBM laptop computer for mobile computing, for user interfaces, data acquisition from field sensors, GPS integration, and wireless ethernet communication in the field. Future versions of this system will be pared down to smaller, more portable units.

\subsection{Construction Project Database}

The project database is essentially a dynamic digital library of everything that will exist on the construction site; joining CAD representation of physical objects together with spatial, time-stamped information concerning their location. The database will contain key project information to aid in the identification and tracking of components, including:

- Component ID number

- User access authorization keys.

- CAD/VRML models.

- Links to material and manufacturer data.

- References to project specifications and drawings.

- Listings of fiducial markings on each component (used to determine the component orientation).

- Chronological history of position and orientation data.

\subsection{Real-Time Kinematic Global Positioning System}

The position and orientation of construction components and sub-assemblies are presently being determined using real-time kinematic (RTK) global positioning system (GPS) equipment. To serve as a base station for data collection, a 1999 Honda TRX$300-4 \times 4$ all-terrain vehicle (ATV) has been instrumented with a GPS receiver and a GPS attitude determination system (ADS). The dual-frequency Ashtec Z-Surveyor GPS receiver provides RTK position accuracy to within $1 \mathrm{~cm}$ horizontal and 1.7 $\mathrm{cm}$ vertical when the receiver is static, and within 3 $\mathrm{cm}$ horizontal and $5 \mathrm{~cm}$ vertical while moving. The Trimble TANS Vector GPS Attitude Determination System uses GPS satellite broadcast signals to determine the three-axis orientation of a platform by calculating the azimuth, pitch, and roll angles of an array of four antennae. For this project, the ADS and GPS receiver are mounted together on the ATV platform to provide complimentary position and orientation data.

An auxiliary roving device is being developed to support the acquisition of position and orientation data for points remote to the GPS/ADS base station on the ATV. By registering the ATV's position and orientation in space to a reference base station, and performing the necessary local coordinate transformations, this device will be capable of capturing the location and attitude of sub-assemblies and components on the site. Ultimately, we intend to reduce the complete system to a backpack-size unit that will make use of alternative 3D positioning systems now under development, in addition to GPS.

\subsection{Wireless Transmission}

We are currently using a RangeLAN2 Ethernet Adapter by Proxim to establish the field data uplink with the construction shack integration computer, which is, in turn, connected via a high speed fiber optic net link (presently ATM) with the remote project database. Current technologies enable computers to establish internet (IP) connections over radio signals, thus maximizing the re-use of existing network infrastructure technology, such as web browsers. However, due to varying protocols and interfaces for databases and other network resources, a middleware layer [4] based on IEEE standard 1278 [5] is being developed at NIST ${ }^{2}$. This layer will provide a common protocol to support data sharing for this project.

\section{TESTBED SET-UP}

A construction lay-down area testbed has been established at NIST to provide an initial means for testing and demonstrating the effectiveness of a prototype tracking system.

\footnotetext{
${ }^{2}$ See the companion paper by Pfeffer \& Lattimer entitled "loward Open Network Data-Exchange Protocols for Construction Metrology and Automation: LiveView"
} 
This testbed contains a representative assortment of construction components including structural steel, pre-cast concrete, bricks, and re-bar. Each component is tagged with a bar code or RF transponder encoding the unique identification number assigned to that object in a testbed database.

A laptop computer serves as host for the sensing hardware (barcode and RFID readers) and software, a construction database, and the web pages which guide users through the component identification process. The database was created using Microsoft Access97, and the web pages were created using Microsoft FrontPage 98 and VisualBasic Script. The web pages, accessed via a web browser such as Internet Explorer 4.1, prompt the user to scan a bar code or RF transponder and then query the database to return information associated with the scanned component, including a 3D model of the object created in SolidWorks97 and exported as a VRML 1.0 file.

We anticipate expanding the system over the coming months to:

(1) Include fiducial markings in the VRML model with user prompts to obtain the real-time position and orientation data at the corresponding points on the component using the previously described roving device with GPS/ADS capabilities. This is a complex topic since every component delivered to the construction site will have a variety of ways in which it can be oriented. At any given time, only a relatively few facets may be accessible for location. The system must be able to prompt the field agent with a unique set of fiducial points to acquire for any anticipated situation during assembly.

(2) Re-locate the project database to a remote server to test the application of wireless communications. We will exercise this utility on a real, $-\$ 6$ million construction project at NIST with the site being approximately $2 \mathrm{~km}$ from the data server; the ground link will be via ATM fiber.

(3) Establish proto-standards for RFID wireless communication packets. Committee 151 [6] of the Construction Industry Institute (CII) on RFID Tagging in Construction has identified this as one of the most significant obstacles to the widespread use of RFID technology on construction sites. Unlike bar codes, the RFID industry today represents a series of information islands, each with their own proprietary, compressed data transmission standards (local to the reader). The situation, further, cannot be easily resolved by simply agreeing to an ID code format, since this hamstrings one of the real benefits of RFID technology: significant $\mathrm{read} / \mathrm{write}$ local data storage on the chip. High end tags now reach capacities of 8 kbits, which can be used to represent everything from valve maintenance records, inspectors signatures, calibration tests, electrical system resistance, hydrostatic pressure expansion data, painting specifications and the like. The categories are nearly endless and encompass every facet of construction from plumbing, to electrical, lighting, fire sensing and suppression, HVAC, metal working, welding, and burning, just to name a few. NIST is presently tasked with investigating the issue to determine if suitable application protocols (AP's) presently exist (e.g. CimSteel, for fabricated steel components, PlantSTEP for process plant piping) and, where none exist, developing industry consortia to develop a common framework.

\section{FUTURE APPLICATIONS}

The prototype testbed tracking system will be evaluated and expanded for full-scale testing: First, at the Building 205 emission control system project at the NIST, Gaithersburg campus, scheduled to breakground winter 1999/2000; then, at the much larger (\$300 million U.S.) Advanced Measurements Laboratory construction project slated to start spring 2001.

\section{REFERENCES}

[1] Finch, E., Flanagan, R. \& Marsh, L, (1996). "Enabling Technologies: a primer on bar coding for construction." The Chartered Institute of Building.

[2] Stukhart, G., and Perce, S. L. (1988). "Construction bar code standards." Proc., $5^{\text {th }}$ Int. Symp. In Robotics in Constr., Japan Society of Civil Engineering, June 6-8, 361370.

[3] Pataky, Craig. "In Black and White: A Barcode Decoder." Circuit Cellar INK, Issue 104, March 1999. [4] Pfeffer, L. and Latimer D., "Toward Open Network Data-Exchange Protocols for Construction Metrology and Automation: LiveView," to be published in the proceedings of the 16th International Symposium on Automation and Robotics in Construction (ISARC 16), Madrid, Spain, September, 1999.

[5] IEEE, "IEEE Std. 1278-1a-1998, Standard for Distributed Interactive Simulations - Application Protocols," IEEE Customer Service, Piscataway, NJ 0855 , (800) 678-1EEE, http://stdbbs.ieee.org/ (refers to 1995 ver.)

[6] CI, 3208 Red River St., Suite 300, Austin, TX 78705-2697 


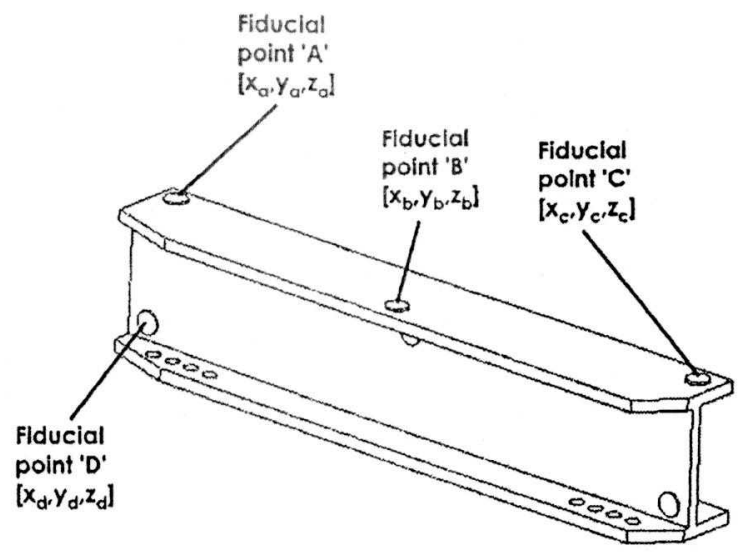

Fig. 1: Typical fabricated steel section with assembly holes on the bottom flange only. The beam is not laterally symmetric either. This situation commonly arises in steel construction, as does the use of unconventional steel material properties, where higher strength, but more flexible, interior members may be used to improve the dynamics of tall buildings by shifting the stiffness outward from the core. All of these practices point to the need for a unique fiducial point labeling system. The fiducial points comprise a necessary and sufficient set of physical locations needed to establish the position and orientation of the component in 3D space regardless of the anticipated access restrictions -- which may change with time. A "smart" fiducial advisory program will prompt a field inspector as to what points are needed and where they are located.

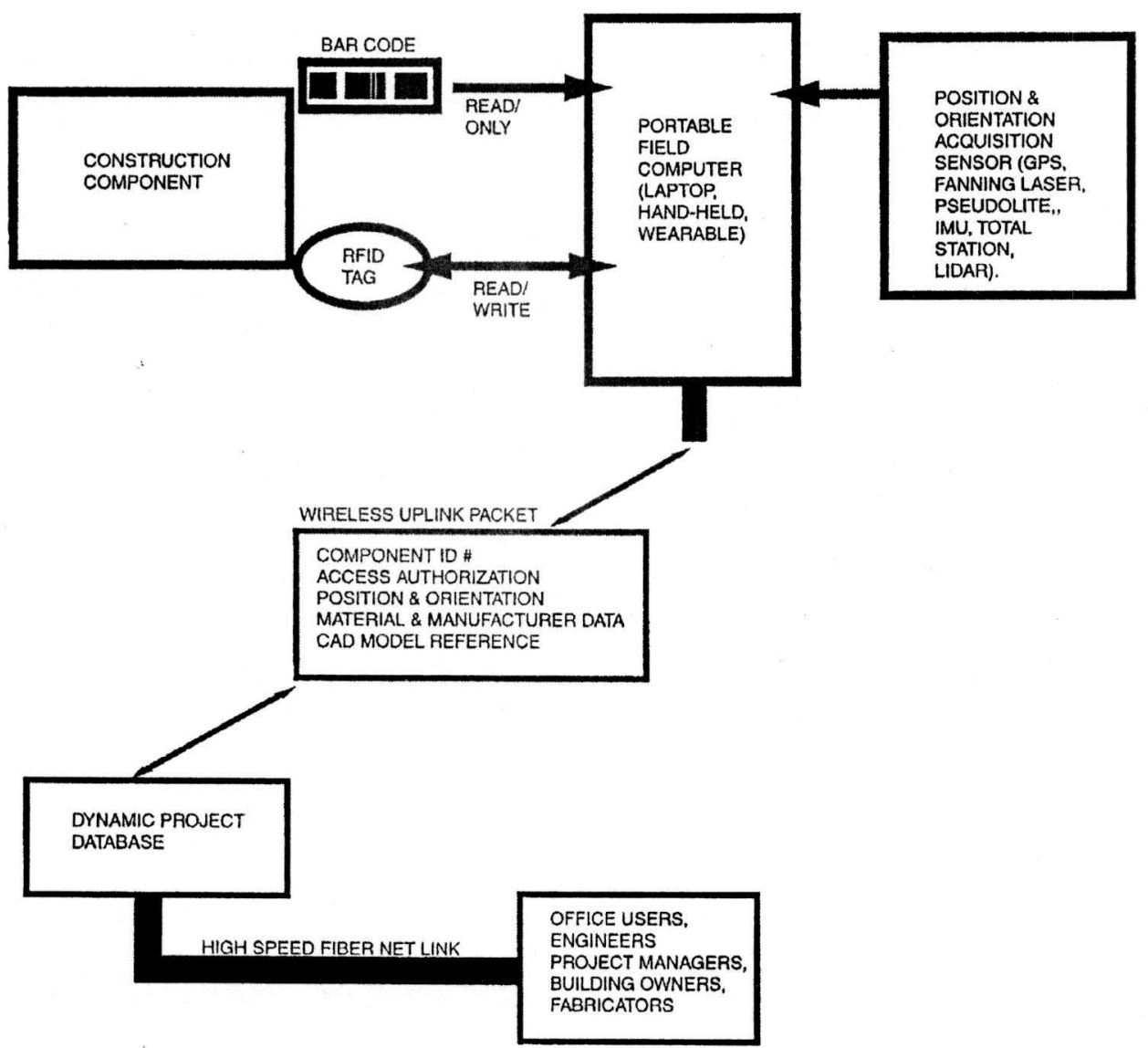

Fig. 2: Schematic of information flow for discrete component tracking. The ficld data logger must be able to interface not only with standard bar codes, but also with proprietary RFID tags, and a host of potential "plug-in" position, attitude, and auxiliary "aiding" sensors. A standard wireless uplink protocol is needed to permit third-party software developers to enter the market and thereby develop specialty plug-ins which access the dynamic database and calculate derivitive quantities useful to contractors and project managers. 


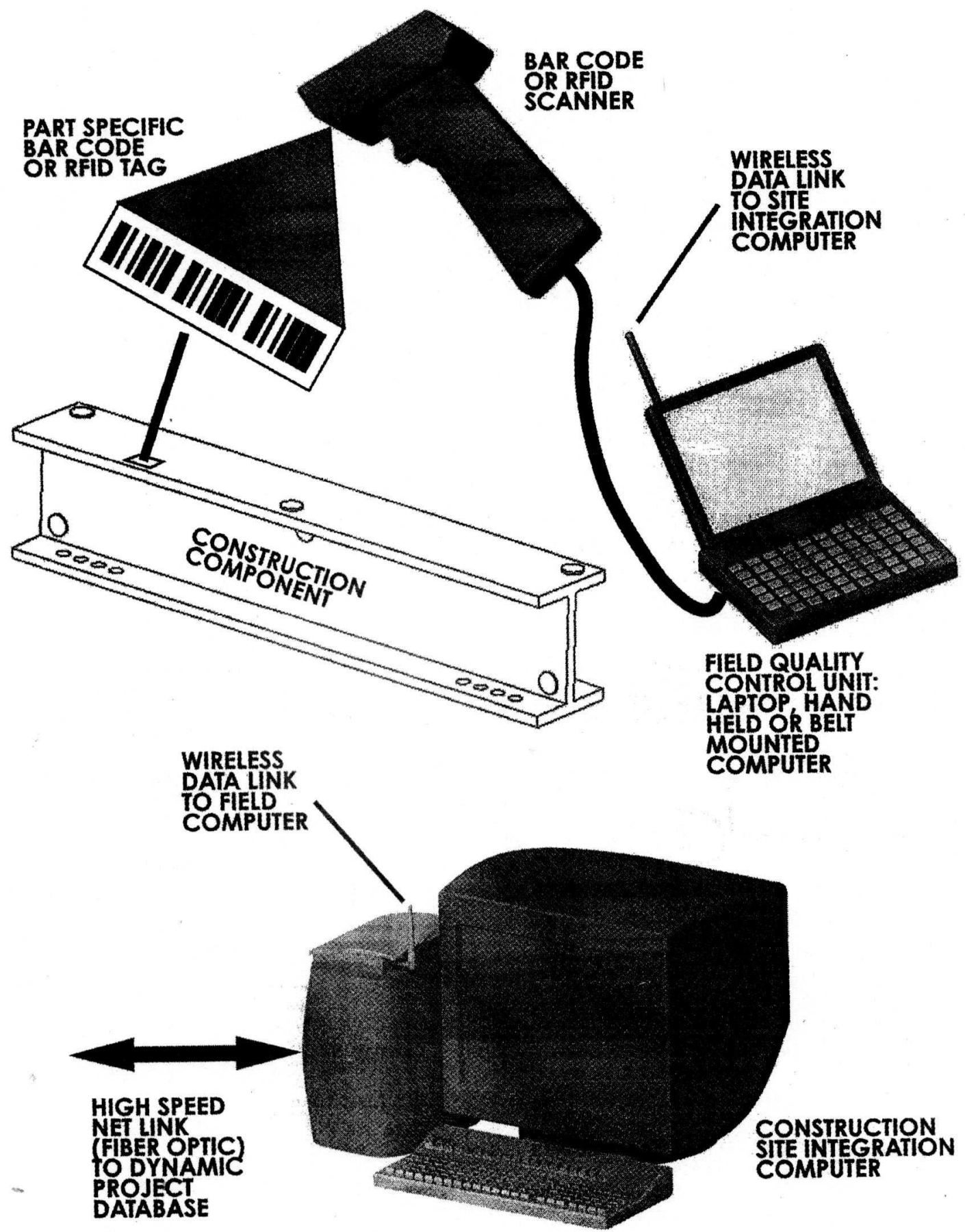

Fig. 3: Some physical hardware used in the NIST construction automation testbed. An Aedex Edge bar code scanner and a Philips HT EV400 Hitag RFID tag and scanner are integrated into an IBM ThinkPad laptop. A Proxim RangeLAN2 PCMCIA plug-in allows for wireless data transfer with a master site integration computer. Presently the site computer is an SGI workstation, equipped with a RangeLAN2 base and running World ToolKit 8 as the 3D simulation system. The integration computer is linked to other labs throughout NIST via an ATM 155 megabit/s fiber. The present database for the discrete component tracking tests is based on MicroSoft Access 97 . The entire system runs through an Internet Explorer 4.1 GUI written in MicroSoft FrontPage 98, driven by a Visual Basic script. The weak link in the system is the wireless LAN, which is limited by both bandwidth and range. 